\title{
Magnetorotational instability in proto-neutron stars
}

\author{
V. Urpin \\ Departament de Física Aplicada, Universitat d'Alacant, Ap. Correus 99, 03080 Alacant, Spain \\ A.F. Ioffe Institute of Physics and Technology, 194021 St. Petersburg, Russia \\ Isaac Newton Institute of Chile, Branch in St. Petersburg, 194021 St. Petersburg, Russia
}

Received 14 July 2009 / Accepted 1 October 2009

\begin{abstract}
Context. Magneto-rotational instability (MRI) has been suggested to lead to a rapid growth of the magnetic field in core collapse supernovae and produce departures from spherical symmetry that are important in determining the explosion mechanism.

Aims. We address the problem of stability in differentially rotating magnetized proto-neutron stars at the beginning of their evolution. Methods. To do this, we consider a linear stability taking into account non-linear effects of the magnetic field and strong gravity.

Results. Criteria for MRI are derived without simplifying assumptions about a weak magnetic field. In proto-neutron stars, these criteria differ qualitatively from the standard condition $\mathrm{d} \Omega / \mathrm{d} s<0$ where $\Omega$ is the angular velocity and $s$ the cylindrical radius. If the magnetic field is strong, the MRI can occur only in the neighbourhood of the regions where the spherical radial component of the magnetic field vanishes. The growth rate of the MRI is relatively low except for perturbations with very small scales which usually are not detected in numerical simulations. We find that MRI in proto-neutron stars grows more slowly than the double diffusive instability analogous the Goldreich-Schubert-Fricke instability in ordinary stars.
\end{abstract}

Key words. stars: neutron - stars: rotation - stars: magnetic field - supernovae: general - instabilities

\section{Introduction}

There is a growing amount of evidence that core-collapse supernovae are asymmetric and that the core-collapse mechanism itself is responsible for the asymmetry (see Buras et al. 2003; Akiyama et al. 2003, for more details). Several possibilities are explored to account for this observed asymmetry. One is associated with the influence of rotation on convection, which seems to be inevitable during the early evolution of proto-neutron stars (PNS). Convective motions in PNSs are very fast $\left(\sim 10^{8}-10^{9} \mathrm{~cm} / \mathrm{s}\right)$ and, therefore, the convective turnover time is short, $\sim 1-10 \mathrm{~ms}$ (see, e.g., Burrows \& Lattimer 1986). Nevertheless, if angular momentum is conserved, the collapsing core can spin up to very short periods $\sim 5-10 \mathrm{~ms}$ and generate strong differential rotation. Such fast rotation modifies convection and makes convective motions anisotropic and constrained to the polar regions (Fryer \& Heger 2000; Miralles et al. 2004). This mechanism is a natural way to create anisotropic energy and momentum transport by convective motions, that only requires that the angular velocity be of the order of the Brunt-Väisälä frequency.

The other possibility to create asymmetry is the effect of jets (see, e.g., Khokhlov et al. 1999; Wheeler et al. 2002). Even though the mechanism of jet formation is still unclear, it seems that MHD jets are common in systems where a central body accretes matter with angular momentum and magnetic field (see, e.g., Meier et al. 2001), and a core-collapse supernova is such a system. Calculations have established that nonrelativistic axial jets originating within the collapsed core can initiate a bipolar asymmetric supernova explosion that is consistent with observations (Hwang et al. 2000).

Another way to generate asymmetry is associated with the magnetic field that can be an important ingredient of the explosion mechanism (Bisnovatyi-Kogan 1971; Kundt 1976). The toroidal magnetic field can be amplified by differential rotation to such high values that it becomes dynamically important (Ardelyan et al. 2005). The effect of the magnetic field on asymmetry of supernovae was considered by Wheeler et al. (2000, 2002) who found that it is possible to produce both a strong toroidal field and an axial jet. Two-dimensional MHD simulations of core collapse indicate that the shape of shock waves and the neutrinosphere can be modified by the effect of the magnetic field (Kotake et al. 2004; Takiwaki et al. 2004).

The possible presence of a magnetic field and differential rotation in a core-collapse supernova favours magnetorotational instability (MRI), which can enhance turbulent transport and amplify the magnetic field. This instability was considered by Akiyama et al. (2003) in the context of core collapse. The authors argued that instability must occur in core collapse and that it has the capacity to produce fields that are sufficiently strong to affect, if not cause, the explosion. Thompson et al. (2005) constructed one-dimensional models, including rotation and magnetic fields, to study the mechanism of energy deposition. They explored several mechanism for viscosity and argue that turbulent viscosity caused by the MRI can be most effective. Numerical simulations provide contradictory conclusions regarding the importance of MRI in core collapse. Moiseenko et al. (2006) claim that MRI has been found in their 2D simulations, and that it is responsible for a strong amplification of the poloidal magnetic flux. However, what these authors call MRI is different to standard MRI considered by Velikhov (1959; see also Balbus \& Hawley 1991). For instance, the instability found by Moiseenko et al. (2006) starts to develop only when the ratio of the toroidal and poloidal fields reaches a value of $\sim$ a few tens. On the other hand, the onset of standard MRI in 2D does not depend on the toroidal field at all. The dependence on the ratio of the toroidal and poloidal field is more typical for Tayler instability (Tayler 1973), which is more relevant to the topology of the 
magnetic field than differential rotation. Therefore, it is possible that Moiseenko et al. (2006) incorrectly identify instability, and MRI does not occur in their simulations. Apart from that, Moiseenko et al. (2006) attributed a rapid growth of the toroidal and poloidal fields to a dynamo driven by the magnetorotational instability. This also rise some doubts because of Cowlings's anti-dynamo theorem which states that an axisymmetric dynamo cannot exist (see, e.g., Shercliff 1965). Two-dimensional simulations of core collapse with a strong magnetic field have been performed by Sawai et al. (2005, 2008). They found that the magnetic field can play an important role in the dynamics of the core only if the poloidal field of the progenitor is strong enough $\left(\sim 10^{12}-10^{13} \mathrm{G}\right)$, but MRI was not seen in the considered models. The magnetic field is amplified mainly by field compression and field wrapping in these simulations. On the contrary, Shibata et al. (2006) claim that they found MRI in their simulations of magnetorotational core collapse in general relativity. These authors paid attention to resolution in order to resolve unstable MRI modes and they claim that amplification of the magnetic field in the considered models is caused by MRI. Note, however, that the poloidal field obtained in their models is of the order of that estimated from conservation of magnetic flux in core collapse, and a more refined analysis is required to determine the mechanism of amplification. Fryer \& Warren (2004) argued that it is difficult to produce magnetic fields in excess of $10^{14}$ $\mathrm{G}$ even if MRI occurs in core collapse because rotation is not sufficiently fast. A detailed study of the magnetorotational core collapse has been performed by Obergaulinger et al. (2006a,b, 2009). The initial magnetic field was purely poloidal in their models with a strength ranging from $10^{10}$ to $10^{13} \mathrm{G}$. Such fields are much higher than those estimated to exist in realistic stellar cores, but the authors wanted to investigate the principal effects of a magnetic field. The initial magnetic field is amplified by differential rotation in these simulations, giving rise to astrong toroidal component. The poloidal component grows mainly by compression during collapse and does not change significantly after core bounce. The authors also found that extended regions exist where the criterion of MRI is satisfied at various epochs. However, the growth rate of this instability is typically too small, except for a few models with a strong initial magnetic field $B=10^{12} \mathrm{G}$.

In this paper, we study the effect of MRI on core-collapse supernova. Since the magnetic field can be sufficiently strong, the criterion of instability is derived taking into account terms depending on the Alfven frequency. We derive the criterion that applies to any rotation profile but a special consideration is made for the case of shellular rotation that often is used to mimic rotation of proto-neutron stars. We address only the axisymmetric instability because numerical simulations of a magnetic corecollapse are usually done in 2D. The main goal of this study is to show that the effect of MRI on proto-neutron stars often is overestimated.

\section{The growth rate of convective and magnetorotational instabilities}

We assume that the initial PNS is restricted by the radius of neutrino sphere. The PNS has a high-entropy mantle, so that the outer part of the star is initially at a relatively large radius. It takes a few tenths of a second for the neutrinos in the highentropy mantle to leak from the star and for the mantle to collapse to the canonical radius. In this paper, we study MRI in relatively deep layers of the PNS where the density is comparable to (or higher than) the nuclear density.
Consider a PNS rotating with angular velocity $\Omega=\Omega(s, z)$; $(s, \varphi, z)$ are cylindrical coordinates. We explore the Boussinesq approximation and assume that the magnetic energy is small compared to the thermal one. In the unperturbed state, the star is in hydrostatic equilibrium,

$$
\frac{\nabla p}{\rho}=\boldsymbol{G}+\frac{1}{4 \pi \rho}(\nabla \times \boldsymbol{B}) \times \boldsymbol{B}, \quad \boldsymbol{G}=\boldsymbol{g}+\Omega^{2} \boldsymbol{s}
$$

where $\boldsymbol{g}$ is the gravity. Generally, the magnetic field $\boldsymbol{B}$ has both toroidal $\boldsymbol{B}_{\varphi}$ and poloidal $\boldsymbol{B}_{\mathrm{p}}$ components. If the field has a component parallel to $\nabla \Omega$, the azimuthal field in the unperturbed state increases with time by winding up the polodal field lines. If the magnetic Reynolds number is large, then

$B_{\varphi}(t)=B_{\varphi}(0)+s\left(\boldsymbol{B}_{\mathrm{p}} \cdot \nabla \Omega\right) t$.

We consider MRI assuming that the basic state is quasistationary. This is justified if the growth rate of the MRI $(\sim \Omega)$ is greater than the inverse time-scale on which the basic state evolves. This inverse time-scale can be estimated as $|\dot{p} / p| \sim$ $s|\nabla \Omega|\left(p_{\mathrm{M}} / p\right) \sim\left(p_{\mathrm{M}} / p\right) \Omega$, where $p_{\mathrm{M}}$ is the magnetic pressure. Therefore, the condition of quasi-stationarity is satisfied if $p_{\mathrm{M}} / p \leq 1$, that is fulfilled in core collapse supernovae.

We consider axisymmetric short-wavelength perturbations with spatial and temporal dependence $\exp (\gamma t-\mathrm{i} \boldsymbol{k} \cdot \boldsymbol{r})$ where $\boldsymbol{k}=\left(k_{s}, 0, k_{z}\right)$ is the wave-vector. Small perturbations will be indicated by a subscript 1 . Then, the linearized MHD equations read in a short-wavelength approximation

$$
\begin{array}{r}
\gamma \boldsymbol{v}_{1}+2 \boldsymbol{\Omega} \times \boldsymbol{v}_{1}+\boldsymbol{e}_{\varphi} s\left(\boldsymbol{v}_{1} \nabla \Omega\right)=\frac{\mathrm{i} \boldsymbol{k} p_{1}}{\rho}+\boldsymbol{G} \frac{\rho_{1}}{\rho} \\
+\frac{\mathrm{i}}{4 \pi \rho}\left[\boldsymbol{k}\left(\boldsymbol{B} \cdot \boldsymbol{B}_{1}\right)-\boldsymbol{B}_{1}(\boldsymbol{k} \cdot \boldsymbol{B})\right],
\end{array}
$$

$\boldsymbol{k} \cdot \boldsymbol{v}_{1}=0$

$\gamma \boldsymbol{B}_{1}=-\mathrm{i} \boldsymbol{v}_{1}(\boldsymbol{k} \cdot \boldsymbol{B})+\boldsymbol{s e}_{\varphi}\left(\boldsymbol{B}_{1} \cdot \nabla \Omega\right)$,

$\boldsymbol{k} \cdot \boldsymbol{B}_{1}=0$,

where $\boldsymbol{e}_{\varphi}$ is the unit vector in the $\varphi$-direction. We neglect kinematic and magnetic viscosities since they are small in PNSs. The term $\propto \nabla \times \boldsymbol{B}$ in Eq. (2) is also neglected. In the short-wavelength approximation, the latter is justified if $B_{\varphi}$ satisfies the inequality $B_{\varphi}<(L / \lambda) B_{\mathrm{p}}$, where $L$ is the length scale of unperturbed quantities and $\lambda=2 \pi / k$.

The matter is assumed to be in chemical equilibrium, thus the density is a function of the pressure $p$, temperature $T$ and lepton fraction $Y$. In the Boussinesq approximation, perturbations of the pressure are small and, therefore, $\rho_{1}$ can be expressed in terms of $T_{1}$ and $Y_{1}$,

$\rho_{1} \approx-\rho \beta\left(T_{1} / T\right)-\rho \delta Y_{1}$,

where $\beta$ and $\delta$ are the coefficients of thermal and chemical expansion; $\beta=-(\partial \ln \rho / \partial \ln T)_{p Y}, \delta=-(\partial \ln \rho / \partial Y)_{p T}$. Since diffusive timescales are long compared to dynamical ones (Miralles et al. 2000), the linearized transport equations read

$\dot{T}_{1}-\boldsymbol{v}_{1} \cdot \Delta \nabla T=0$

$\dot{Y}_{1}+\boldsymbol{v}_{1} \cdot \nabla Y=0$

where $\Delta \nabla T=(\partial T / \partial p)_{s, Y} \nabla p-\nabla T$ is the super-adiabatic temperature gradient. 
The dispersion equation corresponding to Eqs. (2)-(8) is

$\gamma^{4}+b_{2} \gamma^{2}+b_{0}=0$

where

$b_{2}=2 \omega_{\mathrm{A}}^{2}+\omega_{\mathrm{c}}^{2}+q^{2}, \quad b_{0}=\omega_{\mathrm{A}}^{2}\left(\omega_{\mathrm{A}}^{2}+\omega_{\mathrm{c}}^{2}+q^{2}-4 \Omega^{2} k_{z}^{2} / k^{2}\right)$.

Here $\omega_{\mathrm{A}}=(\boldsymbol{k} \cdot \boldsymbol{B}) / \sqrt{4 \pi \rho}$ is the Alfven frequency and

$q^{2}=\left(k_{z}^{2} \Omega_{\mathrm{e}}^{2}-s k_{s} k_{z} \Omega_{z}^{2}\right) / k^{2}, \quad \omega_{\mathrm{c}}^{2}=-\boldsymbol{C} \cdot\left[\boldsymbol{G}-\boldsymbol{k}(\boldsymbol{k} \cdot \boldsymbol{G}) / k^{2}\right]$,

where $C=-(\beta / T) \Delta \nabla T+\delta \nabla Y, \Omega_{\mathrm{e}}$ is the epicyclic frequency, $\Omega_{\mathrm{e}}^{2}=\partial\left(s^{4} \Omega^{2}\right) / s^{3} \partial s$, and $\Omega_{z}^{2}=\partial \Omega^{2} / \partial z$.

Equation (9) has an unstable solution if either $b_{2}$ or $b_{0}$ is negative, or

$2 \omega_{\mathrm{A}}^{2}+\omega_{\mathrm{c}}^{2}+q^{2}<0, \quad \omega_{\mathrm{A}}^{2}+\omega_{\mathrm{c}}^{2}+q^{2}-4 \Omega^{2}\left(k_{z}^{2} / k^{2}\right)<0$.

In the limit of a weak field, these conditions yield

$\omega_{\mathrm{c}}^{2}+q^{2}<0, \quad \omega_{\mathrm{c}}^{2}+q^{2}-4 \Omega^{2}\left(k_{z}^{2} / k^{2}\right)<0$.

The first inequality is the criterion of convection modified by rotation, and the second condition represents the criterion of MRI.

The solution of Eq. (9) is

$\gamma^{2}=-\omega_{\mathrm{A}}^{2}-\frac{1}{2}\left(\omega_{\mathrm{c}}^{2}+q^{2}\right) \pm\left[\frac{1}{4}\left(\omega_{\mathrm{c}}^{2}+q^{2}\right)^{2}+4 \omega_{\mathrm{A}}^{2} \Omega^{2} \frac{k_{z}^{2}}{k^{2}}\right]^{1 / 2}$.

If $\omega_{\mathrm{A}}$ is small compared to the other frequencies, we have

$\gamma_{1,2}^{2}=-\left(\omega_{\mathrm{c}}^{2}+q^{2}\right), \quad \gamma_{3,4}^{2}=-\omega_{\mathrm{A}}^{2}\left[1-\frac{4 \Omega^{2} k_{z}^{2}}{k^{2}\left(\omega_{\mathrm{c}}^{2}+q^{2}\right)}\right]$

The solutions $\gamma_{1,2}$ correspond to buoyant modes that cause convection and are unstable if $\omega_{\mathrm{c}}^{2}+q^{2}<0$. The solutions $\gamma_{3,4}$ describe the magnetorotational modes which can be unstable if the second condition (11) is satisfied.

For the purpose of illustration, we plot in Fig. 1 the dependence of $\gamma^{2}$ on $\omega_{\mathrm{A}} / \Omega$ for the unstable magnetorotational mode. Its growth rate is given by Eq. (12) with the upper sign. Even if stratification is negligible, the growth rate of this mode is typically low and $\gamma \sim \omega_{\mathrm{A}}$ in a weak magnetic field $\left(\omega_{\mathrm{A}}<\Omega\right)$. Stratification can substantially decrease the growth rate and this is seen very well from the figure. For example, the growth rate decreases approximately by a factor of two if $\omega_{\mathrm{c}}^{2} / \Omega^{2}$. At $\omega_{\mathrm{c}}^{2} / \Omega^{2} \approx 1$, stable stratification completely suppresses the magnetorotational instability of the considered perturbations.

In the convective zone where $\omega_{\mathrm{c}}^{2}+q^{2}<0$, the quantity $\omega_{\mathrm{c}}^{2}+$ $q^{2}-4 \Omega^{2}\left(k_{z}^{2} / k^{2}\right)$ is also negative and, hence, $\gamma_{3,4}^{2}<0$. Therefore, the magnetorotational instability does not occur in convectively unstable regions, and these two instabilities are spatially separated if the magnetic field is weak (see also Obergaullinger et al. 2009). The value of $\omega_{\mathrm{c}}$ is of the order of $1-10 \mathrm{~m} \mathrm{~s}^{-1}$ in collapsing cores (see Thompson et al. 2005). Therefore, rotation has an important impact on convection only if $\Omega$ is of the same order of magnitude, $\Omega \approx 1000 \mathrm{rad} / \mathrm{s}$ (Miralles et al. 2004). This value can be reached in PNS if rotation of the progenitor was very fast (Villain et al. 2004). On the contrary, MRI can have an important influence even if rotation is slower.



Fig. 1. The dependence of $\gamma^{2}$ on $\omega_{\mathrm{A}} / \Omega$ for $\Omega \propto 1 / s$ and $k_{z} / k=\sqrt{2} / 2$ and for three values of the ratio $\omega_{\mathrm{c}}^{2} / \Omega^{2}=0.0,0.5$, and 0.8 .

\section{The criteria of magnetorotational instability}

The condition of MRI (second inequality (10)) depends on the direction of $\boldsymbol{k}$ and can be written as follows

$F \equiv \frac{b_{0}}{\omega_{\mathrm{A}}^{2}}=\omega_{0}^{2}+A \frac{k_{z}^{2}}{k^{2}}-D \frac{k_{s} k_{z}}{k^{2}}+E \frac{k_{s}^{2}}{k^{2}}<0$,

where

$A=s \Omega_{s}^{2}+\cos ^{2} \alpha \omega_{\mathrm{A} 0}^{2}+C_{z} G_{z}, \quad E=\sin ^{2} \alpha \omega_{\mathrm{A} 0}^{2}+C_{s} G_{z}$,

$D=s \Omega_{z}^{2}-\sin 2 \alpha \omega_{\mathrm{A} 0}^{2}-C_{s} G_{z}-C_{z} G_{s}$.

In these expressions, we denote

$\omega_{0}^{2}=-\boldsymbol{C} \cdot \boldsymbol{G}, \quad \omega_{\mathrm{A} 0}^{2}=\frac{k^{2} B^{2}}{4 \pi \rho}, \quad \Omega_{s}^{2}=\frac{\partial \Omega^{2}}{\partial s}$

$\alpha$ is the angle between the magnetic field and the rotational axis, $\cos \alpha=B_{z} / \sqrt{B_{z}^{2}+B_{s}^{2}}$.

Since the dependence of $F$ on the direction of $\boldsymbol{k}$ is simple, we can obtain that $F$ reaches its minimum at

$\frac{k_{z}^{2}}{k^{2}}=\frac{1}{2}\left[1 \pm \sqrt{\frac{(A-E)^{2}}{(A-E)^{2}+D^{2}}}\right]$

The value of $F$ corresponding to these $k_{z}^{2} / k^{2}$ yields the following condition of instability

$s \Omega_{s}^{2}+\omega_{0}^{2}+\omega_{\mathrm{A} 0}^{2} \pm \sqrt{D^{2}+(A-E)^{2}}<0$.

By taking the curl of Eq. (1), it can be readily obtained that the condition of hydrostatic equilibrium leads to

$s \Omega_{z}^{2}=\left[\boldsymbol{C} \times \boldsymbol{G}_{B}-\boldsymbol{L}\right]_{\varphi}=C_{z} G_{B s}-C_{s} G_{B z}-L_{\varphi}$,

where

$\boldsymbol{G}_{B}=\boldsymbol{G}+\frac{1}{4 \pi \rho}(\nabla \times \boldsymbol{B}) \times \boldsymbol{B}, \quad \boldsymbol{L}=\nabla \times\left(\boldsymbol{G}_{B}-\boldsymbol{G}\right)$.

We consider only the magnetic field satisfying the condition $L \ll s \Omega_{s}^{2}$ that is approximately equivalent to the requirement 
that the magnetic energy is small compared to the rotational energy. Then, Eq. (17) can be further simplified to obtain

$$
\begin{aligned}
& s \Omega_{s}^{2}+\omega_{0}^{2}+\omega_{\mathrm{A} 0}^{2} \pm\left\{\left(s \Omega_{s}^{2}+\omega_{0}^{2}+\omega_{\mathrm{A} 0}^{2}\right)^{2}\right. \\
& \quad+G_{z}\left[s\left(C_{z} \Omega_{s}^{2}-C_{s} \Omega_{z}^{2}\right)+\omega_{\mathrm{A} 0}^{2}\left(C_{z} \cos 2 \alpha+C_{s} \sin 2 \alpha\right)\right] \\
& \left.\quad-\sin ^{2} \alpha \omega_{\mathrm{A} 0}^{2}\left(\omega_{0}^{2}+s \Omega_{s}^{2}\right)\right\}<0 .
\end{aligned}
$$

The two conditions of instability follow straightforwardly from the above expression:

$$
\begin{aligned}
& s \Omega_{s}^{2}+\omega_{0}^{2}+\omega_{\mathrm{A} 0}^{2}<0, \\
& s G_{z}\left(C_{z} \Omega_{s}^{2}-C_{s} \Omega_{z}^{2}\right)+\omega_{\mathrm{A} 0}^{2}\left[C_{z} G_{z} \cos ^{2} \alpha+C_{s} G_{z} \sin 2 \alpha\right. \\
& \left.\quad+\left(C_{s} G_{s}-s \Omega_{s}^{2}\right) \sin ^{2} \alpha\right]>0 .
\end{aligned}
$$

Conditions (20) and (21) look like the Solberg-Høiland conditions (Tassoul 2000), but with additional terms due to the chemical composition gradients and the magnetic field. If the magnetic field is weak and $\nabla Y=0$ then Eqs. (20) and (21) yield

$$
s \Omega_{s}^{2}+\omega_{0}^{2}<0 ; \quad s G_{z}\left(C_{z} \Omega_{s}^{2}-C_{s} \Omega_{z}^{2}\right)>0 .
$$

The first criterion is similar to the Schwarzschild criterion for convection modified by rotation. However, the Schwarzschild criterion at $B=0$ involves the angular momentum gradient, whereas the criterion of magnetorotational instability depends on the angular velocity gradient, as was noted by Balbus (1995). If rotation is cylindrical with $\Omega_{z}^{2}=0$, the second criterion (22) yields the standard condition of the MRI, $\Omega_{s}^{2}<0$, since $C_{z} G_{z}<0$ in a convectively stable region.

Taking into account that $C_{z} \Omega_{s}^{2}-C_{s} \Omega_{z}^{2}=\left(\boldsymbol{C} \times \nabla \Omega^{2}\right)_{\varphi}$, Eq. (21) can be transformed into

$$
\begin{aligned}
s G_{z}\left|C \| \nabla \Omega^{2}\right| \sin \psi+\omega_{\mathrm{A} 0}^{2}\left[C_{z} G_{z} \cos ^{2} \alpha+C_{s} G_{z} \sin 2 \alpha\right. & \\
& \left.+\left(C_{s} G_{s}-s \Omega_{s}^{2}\right) \sin ^{2} \alpha\right]>0,
\end{aligned}
$$

where $\psi$ is the angle between vectors $C$ and $\nabla \Omega^{2}$. If the Alfven frequency is small, then the criterion of MRI reads

$0>\psi>-\pi$,

and is different from the usually used condition $\Omega_{s}^{2}<0$. Criterion (24) implies that the component of $\nabla \Omega^{2}$ perpendicular to $\boldsymbol{C}$ must have a negative projection on $\boldsymbol{e}_{s}$ for instability whereas the component along $\boldsymbol{C}$ plays no role. In the general case when $\omega_{\mathrm{A} 0}$ is comparable to (or greater than) other frequencies (generally, that is possible for very short wavelengths even if $B$ is weak), criterion (23) can be complicated. For instance, if $\omega_{\mathrm{A} 0} \gg \Omega$, then criterion (23) yields

$C_{z} G_{z} \cos ^{2} \alpha+C_{s} G_{z} \sin 2 \alpha+\left(C_{s} G_{s}-s \Omega_{s}^{2}\right) \sin ^{2} \alpha>0$.

This condition depends on the direction of $\boldsymbol{B}$ and is different from the standard condition $\Omega_{s}<0$. Note, for example, that this condition cannot be satisfied if $B_{s}=0$ because in this case $\sin \alpha=0$ and $C_{z} G_{z}<0$ in a convectively stable region.

\section{Instability in core-collapse supernovae}

The occurence of MRI is sensitive to the rotation profile. It follows from both theoretical modelling and analytic consideration that core collapse of a rotating progenitor results in differential rotation of a protoneutron star (Zwerger \& Müller 1997; Dimmelmeier et al. 2002; Müller et al. 2004; Obergaulinger et al. 2006a,b). Many studies of MRI model rotation of the collapsing core by a shellular profile with $\Omega=\Omega(r)$ where $r$ is the

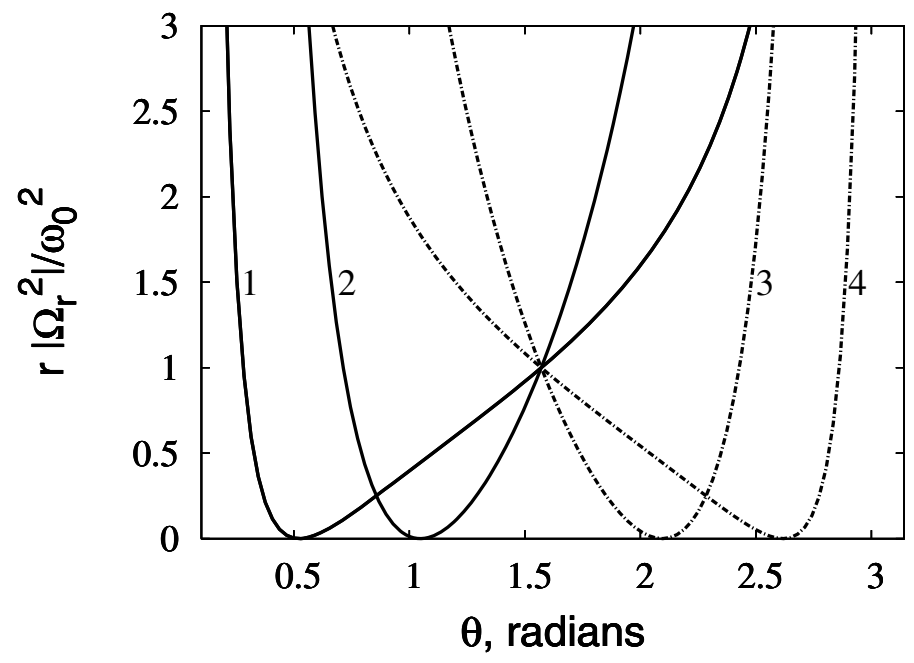

Fig. 2. The dependence of the critical value of $r\left|\Omega_{r}^{2}\right|$ that determines the instability on the polar angle for $\alpha=-\pi / 3$ (curve 1 ). $-\pi / 6(2), \pi / 6$ (3), and $\pi / 3$ (4). The regions above the lines correspond to instability.

spherical radius (see, e.g., Akiyama et al. 2003; Thompson et al. 2005; Sawai et al. 2005). Such rotation can be justified if the progenitor rotates with an angular velocity that depends on $r$ alone (Mönchmeyer \& Müller 1989). Then, if the angular momentum is conserved, it can be shown that rotation of the collapsing core is shellular at the beginning of evolution, at least (see, e.g., Akiyama et al. 2003). However, there arestudies that assume a cylindrical rotation for the initial profile (see, e.g., Obergaulinger et al. 2006a,b).

We assume that the angular velocity is low compared to the Keplerian one and little departure occurs from a spherical geometry. Then, $\boldsymbol{C}$ and $\boldsymbol{G}$ are approximately radial, and we have

$G_{s}=-g \sin \theta, G_{z}=-g \cos \theta, C_{s}=C \sin \theta, C_{z}=C \cos \theta$,

where $\theta$ is the polar angle. The quantity $C$ is negative in a convectively unstable region and positive in a stable region.

Substituting expressions (26) into Eqs. (20), (21), we obtain

$s \Omega_{s}^{2}+\omega_{0}^{2}+\omega_{\mathrm{A} 0}^{2}<0$

$s \omega_{0}^{2} \cos \theta\left(\cos \theta \Omega_{s}^{2}-\sin \theta \Omega_{z}^{2}\right)$

$$
+\omega_{\mathrm{A} 0}^{2}\left[\omega_{0}^{2}(\cos \theta \cos \alpha+\sin \theta \sin \alpha)^{2}+s \Omega_{s}^{2} \sin ^{2} \alpha\right]<0 .
$$

Condition (28) can be satisfied only if the angular velocity is higher than the Brunt-Väisälä frequency which is rather high in core-collapse supernovae. For example, according to calculations by Thompson et al. (2005), the value of $\omega_{0}$ decreases from $\sim 10^{3}$ to $10^{2} \mathrm{~s}^{-1}$ as the radius increases from 50 to $200 \mathrm{~km}$ in the gain region. The angular velocity can be comparable only if the progenitor rotated very rapidly with a period $\sim 2$ s. Such rotation can be achieved only in the most rapidly rotating stars, and our study does not address such stars. We concentrate on condition (28) that can be satisfied for much slower rotation.

If rotation is approximately shellular as is often assumed and $\Omega \approx \Omega(r)$, then we have $\Omega_{s}^{2}=\Omega_{r}^{2} \sin \theta$ and $\Omega_{z}^{2}=\Omega_{r}^{2} \cos \theta$ where $\Omega_{r}^{2}=\partial \Omega^{2} / \partial r$. Then, Eq. (28) can be transformed into

$\omega_{0}^{2} \cos ^{2}(\theta-\alpha)+r \sin ^{2} \theta \sin ^{2} \alpha \Omega_{r}^{2}<0$.

It turns out that stratification strongly suppresses MRI for such a rotation profile because $\omega_{0}^{2}>0$ in convectively stable regions and, typically, $\omega_{0}^{2}>\Omega^{2}$. 
In Fig. 2, we plot the critical value of $r \Omega_{r}^{2}$ that discriminates between stable and unstable regions as a function of $\theta$ for several values of the angle $\alpha$. The region above the corresponding curve is magnetorotationally unstable. For any given $\alpha$ and negative $\Omega_{r}^{2}$, there exists a range of $\theta$ where the instability can arise. If $r \Omega_{r}^{2}$ is greater than (or comparable to) $\omega_{0}^{2}$ then the instability occurs over a rather wide range of $\theta$. If $r\left|\Omega_{r}^{2}\right| \ll \omega_{0}^{2}$ (which is more typical for PNSs) then the instability arises only in a very narrow range of $\theta$. This dependence can be easily understood from Eq. (29). Even if $\Omega_{r}^{2}<0$, MRI occurs only in those regions where the first term on the 1.h.s. of Eq. (29) is small. This occurs in the neighbourhood of the line

$\theta=\alpha \pm \pi / 2$.

Condition (30) implies that the field line is perpendicular to the radius and, hence, at any magnetic topology, the instability occurs only near the region where $B_{r}=0$. How extended this region is depends on the relation between $\omega_{0}^{2}$ and $r \Omega_{r}^{2}$.

There is no generally accepted point of view regarding topology of the magnetic field in core-collapse supernovae. For illustration, we consider the simplest configuration with

$B_{r}=f(r)\left(1-3 \cos ^{2} \theta\right), \quad B_{\theta}=F(r) \sin \theta \cos \theta$,

where $f$ and $F$ are functions of the spherical radius that satisfy the divergence-free condition $F=\mathrm{d}\left(r^{2} f\right) / r \mathrm{~d} r$. This field can be matched to the dipole component outside the star (see, e.g., Urpin et al. 1994). Generally, the field can have a more complex geometry but a simple model (31) allows us to show qualitatively how the magnetic topology infuences the region of MRI. We have for the magnetic field (31)

$$
\begin{aligned}
& \sin \alpha=\frac{\boldsymbol{e}_{s} \cdot \boldsymbol{B}}{B_{p}}=\frac{\sin \theta}{B}\left[f\left(1-3 \cos ^{2} \theta\right)+F \cos ^{2} \theta\right], \\
& \cos \alpha=\frac{\boldsymbol{e}_{z} \cdot \boldsymbol{B}}{B_{p}}=\frac{\cos \theta}{B}\left[f\left(1-3 \cos ^{2} \theta\right)-F \sin ^{2} \theta\right] .
\end{aligned}
$$

Substituting these expressions into Eq. (29), we obtain

$\omega_{0}^{2}\left(1-3 \cos ^{2} \theta\right)^{2}+r \Omega_{r}^{2} \sin ^{4} \theta\left[1-(1-x) \cos ^{2} \theta\right]^{2}<0$,

where $x=\mathrm{d} \ln f / \mathrm{d} \ln r$. Obviously, this inequality can be satisfied only if the angular velocity decreases with $r$. If $\Omega_{r}^{2}<0$, then criterion (33) is always fulfilled at $\cos \theta \approx 1 / \sqrt{3}$ (or at $\theta \approx \theta_{0}=54^{\circ}$ ) except for the case $x=-2$. Hence, some region in the neighbourhood of this polar angle can also be unstable, and the opening angle of the unstable region depends on the ratio $\left|r \Omega_{r}^{2} / \omega_{0}^{2}\right|$. For example, if this ratio is small then MRI occurs within the cone $\theta_{0}+\Delta \theta>\theta>\theta_{0}-\Delta \theta$, where

$\Delta \theta \approx \frac{|2+x|}{3 \sqrt{2}} \frac{\sqrt{\left|r \Omega_{r}^{2}\right|}}{\omega_{0}}$.

\section{Conclusion}

Interest in MRI in core collapse supernova is due to the fact that it can generate a strong magnetic field and produce significant departures from spherical symmetry. These departures are crucial for the explosion mechanism. In the present paper, we have derived criteria of MRI in proto-neutron stars taking into account the effect of a non-axial magnetic field. Criteria have been obtained in a form analogous to the Solberg-Høiland criteria but including terms containing the magnetic field. It turns out that the criterion of MRI in proto-neutron stars can differ from the standard condition $\partial \Omega / \partial s<0$ even in a weak magnetic field due to strong gravity and the gradient of the lepton fraction. If the Alfven frequency $\omega_{\mathrm{A} 0}$ is small compared to the angular velocity then the instability occurs in the region where the component of $\nabla \Omega^{2}$ perpendicular to $C=-(\beta / T) \Delta \nabla T+\delta \nabla Y$ has a negative projection on $\boldsymbol{e}_{s}$ whereas the component $\nabla \Omega^{2}$ parallel to $\boldsymbol{C}$ is unimportant for instability. In the case of slow rotation, when departures from sphericity are small $\left(g \gg s \Omega^{2}\right)$, the criterion reduces to a simple inequality $\partial \Omega / \partial \theta<0$ (see, e.g., Urpin 1996). For instance, shellular rotation with $\Omega=\Omega(r)$ which is often used in modelling proto-neutron stars does not satisfy this condition. Therefore, MRI does not occur if the proto-neutron star rotates shellularly and the wavelength of perturbations is such that the Alfven frequency is smaller than $\Omega$. Only detailed calculations of rotational core collapse can give the answer to whether the condition of MRI is fulfilled in proto-neutron stars and, generally, this answer should depend on rotation of the progenitor. Note that the velocity of unstable perturbations is approximatelly perpendicular to the radius because gravity strongly suppresses motion in the radial direction and, likely, the radial turbulent transport should be suppressed when the instability saturates.

However, MRI can arise in proto-neutron stars even if the neccesary condition that determines the onset of instability in a weak field is not fullfied. This occurs if the magnetic field is very strong or the wavelength of perturbations is small such that $\omega_{\mathrm{A} 0}>\Omega$. The latter inquality is equivalent to

$\lambda<\lambda_{\mathrm{c}}=2 \pi B / \Omega \sqrt{4 \pi \rho} \approx 1.8 \times 10^{3} B_{13} \Omega_{3}^{-1} \rho_{14}^{-1 / 2} \mathrm{~cm}$,

where $\lambda=2 \pi / k$ is the wavelength, $B_{13}=B / 10^{13} \mathrm{G}, \Omega_{3}=$ $\Omega / 10^{3} \mathrm{~s}^{-1}, \rho_{14}=\rho / 10^{14} \mathrm{~g} / \mathrm{cm}^{3}$. For perturbations, satisfying condition (35), the instability can occur if $\Omega_{s}<0$ but only in the neighbourhood of the cone (or cones depending on the field topology) in which the radial component of $\boldsymbol{B}$ is vanishing. The opening angle of the unstable region around this cone is of the order of $\Omega / \omega_{0}$ (see Eq. (34)). Note that unstable motion in this case are perpendicular to the radius as well since gravity suppresses radial motions. The reason why the instability is not entirely suppressed even by a very strong magnetic field is qualitatively very simple. Perturbations with $\boldsymbol{k}=\left(k_{r}, 0,0\right)$ cannot be suppressed by gravity, and such perturbations do not feel the stabilizing influence of the magnetic field in the region where $B_{r} \approx 0$ as well, because this influence is proportional to the Alfven frequency $(\propto \boldsymbol{k} \cdot \boldsymbol{B})$.

It appears that the importance of MRI in core collapse can be overappreciated since its growth rate is relatively low and reaches the value $\sim \Omega$ only for perturbations with a wavelength $\sim \lambda_{\mathrm{c}}$ (see Eq. (35)). Apart from this, gravity strongly suppresses development of any perturbations with non-radial wavevectors. Only perturbations with $k \approx k_{r}$ can be unstable but hydrodynamic motion for such perturbations has a small radial component and turbulent transport should be inefficient radially. Also, it is possible that MRI occurs only in not very extended regions of the proto-neutron star that may diminish substantially its effect on core collapse. Note that since the wavelength $\lambda_{\mathrm{c}}$ is small, one needs a very high resolution in numerical simulations to see the most rapidly growing modes (Obergaulinger et al. 2009). Perturbations with longer $\lambda$ grow substantially slower.

Our conclusion is in contrast to the results obtained by Masada et al. (2007) who considered axisymmetric and nonaxisymmetric magnetorotational instability of PNSs taking into account dissipative processes. These authors also used a local approximation in the stability analysis. In the local analysis, however, the shape of perturbations is assumed to be unchanged 
and, therefore, this approach applies only if the growth rate of instability is greater than the rate with which perturbations change their shape. In the case of MRI, the growth rate is smaller than (or, at maximum, comparable to) $s|\nabla \Omega|$ whereas perturbations change their shape because of differential rotation with a rate $\sim s|\nabla \Omega|$ (see, e.g., Balbus \& Hawley 1992, for details). Therefore, the results obtained for a nonaxisymmetric MRI in the local approximation raise some doubts. As far as axisymmetric instability is concerned, Masada et al. (2007) were confused when identifying different modes in the dispersion equation. In the dissipative case, they obtained a dispersion equation of the seventh order which describes seven different modes. Certainly, different modes can be unstable in different conditions, but the authors considered only one criterion (Eq. (32) of their paper). Unfortunately, this criterion does not correspond to MRI but describes a secular instability that is a magnetic analogy of the well known Goldreich-Schubert-Fricke (GSF) instability. The magnetic analogy of this instability was first considered by Urpin (2006) for the case of ordinary stars, and Masada et al. (2007) obtained the criterion of the same instability modified for the conditions of PNSs. In contrast to MRI, this instability is dissipative and disappears if diffusive coefficients go to zero. Stratification has a weak impact on this dissipative instability, and it can arise in PNSs. Note, however, that diffisive processes can reduce the stabilizing influence of stratification on the MRI as well.

As was noted by Masada et al. (2007), diffusion of heat and leptons can influence buoyancy if the characteristic diffusion timescale is shorter than the buoyant frequency $\omega_{0}$. Since the diffusion timescale is determined mainly by lepton diffusion which is slower than thermal diffusion, this condition is equivalent to $\xi k^{2} \geq \omega_{0}$ where $\xi$ is the coefficient of lepton diffusivity. In deep layers with a density $\sim 10^{14} \mathrm{~g} / \mathrm{cm}^{3}$, the value of $\xi$ is $\sim 3 \times 10^{7} \mathrm{~cm}^{2} \mathrm{~s}^{-1}$ (see Eq. (45) by Masada et al. 2007). Hence, diffusion begins to suppress the stabilizing effect of stratification when the wavelength of perturbations is shorter than $\sim 2 \pi \sqrt{\xi / \omega_{0}} \sim 10^{3} \mathrm{~cm}$ (we suppose $\omega_{0} \sim 10^{3} \mathrm{~s}^{-1}$ ). The effects considered in this paper are important for longer wavelengths for the standard pulsar magnetic field.

As was mentioned above, MRI is not the only instability caused by differential rotation in proto-neutron stars. The analogy of the GSF dissipative instability can occur in both magnetic and non-magnetic PNSs. In non-magnetic stars, this instability arises if the angular velocity depends on the vertical coordinate $z$ (Urpin 2007), and the shellular rotation is a particular case of such rotation. Since neutrino transport in PNSs is much more efficient than radiative transport in ordinary stars, the dissipative instability can be rather fast. If $\Omega_{z}=\partial \Omega / \partial z \sim \Omega / s$, the condition of the instability reads

$\frac{\left|s \Omega_{z}\right|}{\sqrt{\Omega_{\mathrm{e}}^{2}}} \sim \Omega \gg 2 \omega_{0} \sqrt{\frac{v}{\kappa_{\mathrm{T}}}}$,

where $v$ and $\kappa_{\mathrm{T}}$ are the coefficients of viscosity and thermal diffusivity. The difference to the standard criterion of GSF instability $(\partial \Omega / \partial z \neq 0)$ is caused by the more complex character of neutrino trancport that involves diffusion of both heat and the lepton fraction. This instability can arise soon after collapse even in dense central regions of the star where convection does not occur. It turns out that in all cases, when dissipative instability arises, its growth rate is higher than that of MRI. Indeed, the maximum growth rate of MRI is $\sim \Omega$ and can be reached for perturbations with wavelength $\sim \lambda_{\mathrm{c}}$. The growth rate of the dissipative instability is approximately $\kappa_{\mathrm{T}} k^{2}\left(\Omega / \omega_{0}\right)^{2}$. The maximum wavevector for which the instability occurs is $\sim \sqrt{\Omega / v}$. For perturbations with such wavevector, the growth rate is of the order of $\Omega\left(\kappa_{\mathrm{T}} / v\right)\left(\Omega / \omega_{0}\right)^{2}$ and is always higher than the growth rate of MRI if condition (36) is satisfied. Therefore, dissipative instability seems to be more efficient in proto-neutron stars than MRI.

\section{References}

Akiyama, S., Wheeler, J. C., Meier, D., \& Lichtenstadt, I. 2003, ApJ, 584, 954 Ardelyan, N., Bisnovatyi-Kogan, G., \& Moiseenko, S. 2005, MNRAS, 359, 333 Balbus, S. 1995, ApJ, 453, 380

Balbus S., \& Hawley J. 1991, ApJ, 376, 214

Balbus S., \& Hawley J. 1992, ApJ, 400, 610

Bisnovatyi-Kogan, G. 1971, Sov. Astron., 14, 652

Buras, R., Rampp, M., Janka, H.-Th., \& Kifonidis, K. 2003, PRL, 90, 1101

Burrows, A., \& Lattimer, J. M. 1986, ApJ, 307, 178

Dimmelmeier, H., Font, J. A., \& Müller, E. 2002, A\&A, 393, 523

Fryer, C. L., \& Heger, A. 2000, ApJ, 541, 1033

Fryer, C. L., \& Warren M. 2004, ApJ, 601, 391

Hwang, U., Holt, S., \& Petre, R. 2000, ApJ, 537, L119

Khokhlov, A., Höflich, P., Oran, E. S., et al. 1999, ApJ, 524, L107

Kotake, K., Sawai, H., Yamada, S., \& Sato, K. 2004, ApJ, 608, 391 Kundt, W. 1976, Nature, 261, 673

Masada, Y., Sano, T., \& Shibata, K. 2007, ApJ, 655, 447

Meier, D., Koide, S., \& Uchida, Y. 2001, Science, 291, 84

Miralles, J. A., Pons, J. A., \& Urpin, V. 2000, ApJ, 543, 1001

Miralles, J. A., Pons, J. A., \& Urpin, V. 2002, ApJ, 574, 356

Miralles, J. A., Pons, J. A., \& Urpin, V. 2004, A\&A, 420, 245

Moiseenko, S., Bisnovatyi-Kogan, G., \& Ardelyan, N. 2006, MNRAS, 370, 501

Mönchmeyer, R., \& Müller, E. 1989, in Timing Neutron Stars, ed. H. Ögelman,

\& E. P. J. van den Heuvel, NATO ASI Ser. C, 262 (Dordrecht: Kluwer), 549

Müller, E., Rampp, M., Buras, R., Janka, H.-T., \& Shoemaker, D. 2004, ApJ, 603,221

Obergaulinger, M., Aloy, M. A., \& Müller, E. 2006a, 450, 1107

Obergaulinger, M., Aloy, M. A., Dimmelmeier, H., \& Müller, E. 2006b, 457, 209

Obergaulinger, M., Cerda-Duran, P., Müller, E., \& Aloy, M. A. 2009, A\&A, 498, 241

Sawai, H., Kotake, K., \& Yamada, S. 2005, ApJ, 631, 446

Sawai, H., Kotake, K., \& Yamada, S. 2008, ApJ, 672, 465

Shercliff, J. A. 1965, A textbook on magnetohydrodynamics (Oxford: Pergamon) Shibata, M., Liu, Y. T., Shapiro, S., \& Stephen, B. 2006, PRD, 74, 4026

Takiwaki, T., Kotake, K., Nagataki, S., \& Sato, K. 2004, ApJ, 616, 1086

Tassoul, J.-L., 2000, In Stellar Rotation (Cambridge: Cambridge Univ. Press)

Tayler, R. 1973, MNRAS, 161, 365

Thompson, T., Quataert, E., \& Burrows, A. 2005, ApJ, 620, 861

Urpin, V. 1996, MNRAS, 280, 149

Urpin, V. 2006, A\&A, 447, 285

Urpin, V. 2007, A\&A, 469, 639

Urpin, V., Chanmugam, G., \& Sang, Y. 1994, ApJ, 433, 780

Velikhov E. 1959, Sov. Phys. JETP, 9, 995

Villain, L., Pons, J., Cerdá-Durán, P., \& Gourgoulhon, E. 2004, A\&A, 418, 283

Wheeler, J. C., Meier, D. L., \& Wilson, J. R. 2002, ApJ, 568, 807

Wheeler, J. C., Yi, I., Höflich, P., \& Wang, L. 2000, ApJ, 537, 810

Zwerger, T., \& Müller, E. 1997, A\&A, 320, 209 